\title{
Análisis biomecánico in vitro de la desmotomía de los ligamentos suspensorios del navicular equino
}

\author{
In vitro biomechanical analysis of the desmotomy of the suspensory \\ navicular ligaments of the equine
}

\author{
Leonardo Gaona-Urueña ${ }^{1,3}$, Daniel I. Oliveros Wilches ${ }^{2}$, \\ Jorge Alexander León-González ${ }^{2}$
}

\section{ReSUMEN}

El objetivo del estudio fue diseñar un modelo biomecánico del dígito del equino y su uso en el análisis in vitro de la desmotomía de los ligamentos suspensorios del navicular. Se seleccionaron 10 dígitos anteriores ortopédicamente sanos de caballos criollos, los cuales fueron adaptados a un laboratorio para procedimientos biomecánicos para determinar la tensión de los tendones flexores digitales superficial y profundo $\left(\mathrm{T}_{\text {TFDS }}\right.$ y $\mathrm{T}_{\text {TFDP }}$ ) y los cambios biomecánicos del hueso navicular. Se obtuvieron 86 datos por dígito antes del procedimiento quirúrgico y 86 datos después del procedimiento quirúrgico. Se determinó la fuerza sobre el hueso navicular por cada dígito, fuerza sobre el hueso navicular entre los dígitos y la fuerza sobre el hueso navicular entre tiempos quirúrgicos (TQXs). Se observó poca variabilidad y nula diferencia significativa entre las repeticiones experimentales de cada dígito, así como similitud estadística de los TQXs de la mayoría de los dígitos; sin embargo, se evidenciaron diferencias significativas al comparar los TQXs de tres dígitos. El modelo de dinámica inversa permitió establecer la tensión de los tendones flexores digitales y la fuerza sobre el hueso navicular antes y después de la desmotomía de los ligamentos suspensorios del navicular, sin que se observase cambios de fuerza significativos.

Palabras clave: biomecánica; eje podo falángico; navicular

\footnotetext{
${ }^{1}$ Facultad de Medicina Veterinaria, Universidad Pedagógica y Tecnológica de Colombia, Tunja, Boyacá, Colombia

${ }^{2}$ Universidad de Ciencias Aplicadas y Ambientales, Bogotá DC, Colombia

${ }^{3}$ E-mail: leonardogaona@hotmail.com
}

Recibido: 20 de diciembre de 2017

Aceptado para publicación: 26 de septiembre de 2018 
The objective of this study was to design a biomechanical model of the equine digit and its use in the in vitro analysis of the desmotomy of the suspensory ligaments of the navicular. Ten orthopedically healthy anterior digits of Creole horses were collected and adapted to a laboratory for biomechanical procedures to determine the tension of the superficial and deep digital flexor tendons $\left(\mathrm{T}_{\text {TFDS }}\right.$ and $\left.\mathrm{T}_{\text {TFDP }}\right)$ and the biomechanical changes of the navicular bone. In total, 86 data were obtained by digit before the surgical procedure and 86 data after the surgical procedure. The force on the navicular bone, the force on the navicular bone between the digits and the force on the navicular bone between surgical times (TQXs) was determined by each digit. Little variability and none significant difference between the experimental repetitions of each digit was observed, as well as statistical similarity of the TQXs of most of the digits; however, significant differences were found when comparing the three-digit TQXs. The inverse dynamic model allowed establishing the tension of the digital flexor tendons and the force on the navicular bone before and after the desmotomy of the suspensory ligaments of the navicular, and none significant force changes were observed.

Key words: biomechanics; phalangeal axis; navicular

\section{INTRODUCCIÓN}

El síndrome del navicular es una alteración claudogénica bilateral, principalmente de miembros anteriores en equinos, que se presenta en forma crónica y progresiva. Esta patología se considera como la responsable de alrededor de un tercio del total de las cojeras crónicas que afectan los miembros anteriores en equinos (Stashak, 2003).

Varias hipótesis se postulan como causa del síndrome del navicular, pero ninguna se considera un factor etiológico definitivo. Una de estas teorías propone como principal factor etiológico del síndrome del navicular la mala conformación y desequilibrio del pie (Pool et al., 1989). Es por ello que se recomienda la desmotomía de los ligamentos suspensorios del hueso del navicular para tratar la claudicación que se origina (Watkings et al., 1993; Bell et al., 1996). No obstante, en distintas investigaciones de seguimiento posquirúrgico de este tratamiento, se evidencia un porcentaje de efectividad poco homogéneo (Wright, 1986; Watkings et al., 1993; Wright, 1993; Bell et al., 1996).
Una alternativa para la evaluación clínica de claudicaciones es el empleo de técnicas de cinética o cinemática para evaluar los efectos de los procedimientos diagnósticos o terapéuticos en la ortopedia equina (Back y Clayton, 2013). Estudios biomecánicos mediante el análisis de dinámica inversa se usan para evaluar los efectos de la desmotomía del ligamento accesorio del tendón del músculo flexor digital profundo. Alteraciones en la locomoción evidenciadas en estos estudios fueron indicativos claros de la pérdida de la función biomecánica (Buchner et al., 1996; Berckel et al., 1998).

Debido a que no existen estudios previos que incluyan la biomecánica quirúrgica del hueso navicular en condiciones in vitro, el objetivo del presente trabajo fue analizar in vitro el efecto de la desmotomía de los ligamentos suspensorios del navicular, empleando un modelo biomecánico experimental del dígito del equino, a partir de un modelo de transición física de fuerzas. 


\section{Materiales y Métodos}

El estudio fue presentado ante el comité de Ética de la Facultad de Ciencias Agropecuarias de la Universidad de la Salle y fue avalado en la reunión del día 24 de mayo del 2014, registrada en el acta con el consecutivo $\mathrm{N}^{\circ} .92$.

\section{Diseño del Estudio}

Diez dígitos anteriores de equinos se sometieron in vitro a las fuerzas y cargas que experimentan durante la fase de estación y comienzo del movimiento a nivel podotroclear, para medir y comparar la tensión del tendón flexor digital profundo $\left(\mathrm{T}_{\mathrm{TFDP}}\right)$ sobre el hueso navicular, antes y después de una desmotomía de los ligamentos suspensorios del navicular.

\section{Materiales Anatómicos}

Se colectaron dígitos de las extremidades anteriores de 10 caballos (Equus caballus) criollos colombianos entre 3 y 20 años de edad, con masa corporal promedio de $345 \mathrm{~kg}$, sacrificados en una planta de beneficio comercial de la Sabana de Bogotá. Los caballos carecían de lesiones ortopédicas ante mortem y post mortem. Los dígitos se cortaron a nivel del tercio medio del radio, se envolvieron en papel vinipel industrial y se congelaron a $-20{ }^{\circ} \mathrm{C}$ hasta el desarrollo de la prueba in vitro (1 a 3 días).

\section{Materiales Electrónicos}

La fuerza del tendón flexor digital profundo sobre el hueso navicular se midió utilizando un aparato electrónico que incluía un sensor de fuerza Flexiforce ${ }^{\circledR}$ modelo A201, conectado a una tarjeta de circuito impreso Arduino UNO (Atmel®) y su respectivo entorno de programación. El sensor variaba su resistencia eléctrica cuando el tendón flexor digital profundo y el hueso navicular aplicaban presión simultáneamente sobre el área sensible del mismo. Para evitar inestabilidad en la resistencia del sensor, asociada a las superficies irregulares que entraban en contacto, se acondicionaron dos placas metálicas redondas de $1.52 \mathrm{~cm}$ de diámetro y 1 $\mathrm{mm}$ de espesor que cubrían el sensor por ambas caras (Figura 1). Los valores de fuerza obtenidos se registraron cada segundo en una tabla de Excel 2010.

\section{Prototipo Mecánico de Ensayos in vitro}

Para la caracterización mecánica se empleó un aparato diseñado para la ejecución y medición de fuerzas y cargas sobre el dígito del equino. Este prototipo permitió acoplar verticalmente el dígito utilizando un tornillo intramedular que lo fijaba y estabilizaba. Por otro lado, unas guayas metálicas se unieron a los tendones flexores por su extremo proximal y estas a su vez a tensores que conectaban con dinamómetros. Los dinamómetros usados fueron de tipo electrónico con capacidad para $300 \mathrm{~kg}$, y los de tipo mecánico con capacidad para $100 \mathrm{~kg}$. Finalmente, el dedo se apoyaba sobre una báscula mecánica con capacidad de $130 \mathrm{~kg}$. Para aplicar la fuerza gravitacional análoga al peso soportado por un dígito, se utilizó un gato mecánico de tijera de $800 \mathrm{~kg}$ que se ubicaba sobre la plataforma móvil del prototipo (Figura 2).

\section{Consideraciones Mecánicas del Biomodelo}

Se consideró necesario eliminar el efecto biomecánico del aparato estático pasivo del musculo interóseo y la cabeza carpinana del flexor digital profundo para evaluar solamente la fuerza de los tendones flexores digitales superficial y profundo. Para lo anterior, se propuso un modelo de transición estática a dinámica. Dicho modelo determina el balance de fuerzas en los sistemas físicos en equilibrio, estático y dinámico, y consta de tres fases biomecánicas: 1) fase de condición estática, 2) fase de transición y 3) fase de condición dinámica.

La fase de condición estática se refiere al punto donde las fuerzas del aparato estático pasivo se encargan de darle sostén al dígito del 

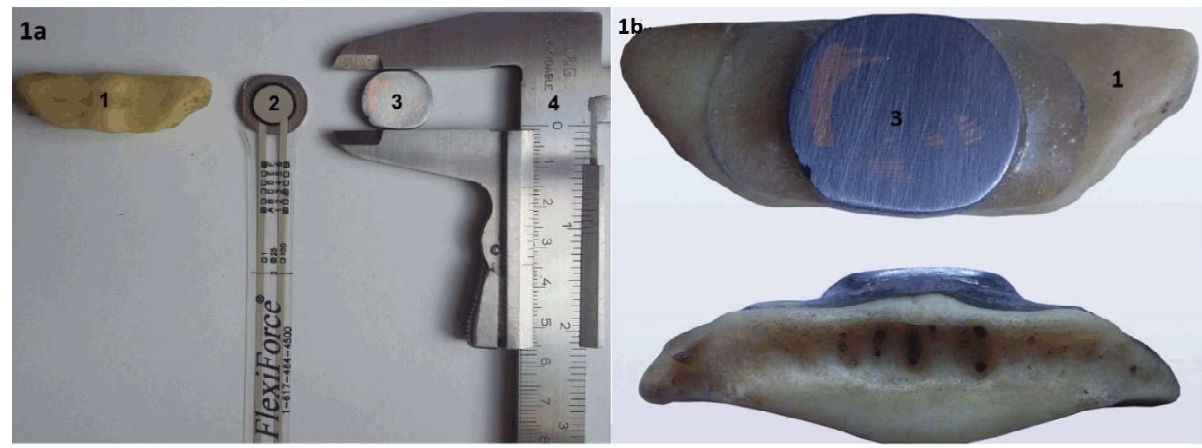

Figura 1. Componentes físicos del sistema de medición de fuerza del tendón flexor digital profundo sobre el hueso del navicular. (1a) Materiales del sistema de medición. (1b) Detalle de la placa metálica ubicada sobre la superficie flexora del hueso navicular 1. Superficie flexora del hueso navicular; 2. Sensor de fuerza Flexiforce ${ }^{\circledR}$ modelo A201 (ubicado debajo la placa metálica); 3. Placa metálica estabilizadora del sensor;

4. Calibrador de diámetro y espesor de placa metálica

equino manteniendo el eje podofalángico constante y en estación a $45^{\circ}$, sin intervención de fuerzas sobre los tendones flexores digitales. En este punto la fuerza de los tendones flexores digitales es $0 \mathrm{kgf}$. La fase de transición se refiere al punto donde diferentes estructuras generadoras de fuerza originan un intercambio de energía y a su vez el inicio de la condición dinámica. En este punto, la energía elástica del ligamento suspensorio del menudillo y la cabeza carpiana del músculo flexor digital profundo comienzan a perder su potencial de energía elástica. Si este proceso de intercambio energético continúa en el tiempo, casi que simultáneamente se dará comienzo a la generación de energía por parte de los músculos flexores digital superficial y profundo, dando paso a la fase dinámica del modelo de transición. La Figura 3 muestra como una condición estática puede dar origen a una condición dinámica.

Es importante anotar que debido a la elongación natural de las fibras tendinosas sometidas a una tensión (Clayton, 2004; Nordin y Frankel, 2013) durante cada experimento, fue necesario ajustar constantemente los tensiómetros para mantener estable la

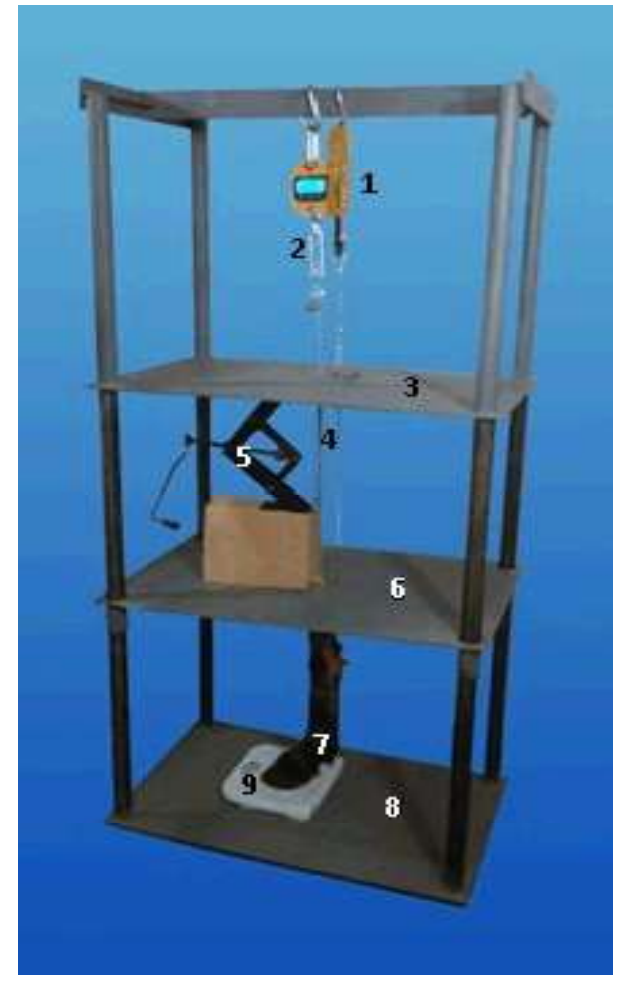

Figura 2. Prototipo de ensayos biomecánicos: 1. Dinamómetro; 2. Tensiómetro; 3. Plataforma fija superior; 4. Guayas; 5. Gato mecánico de tijereta; 6. Plataforma móvil; 7. Dígito del equino; 8. Plataforma fija inferior; 9. Báscula 


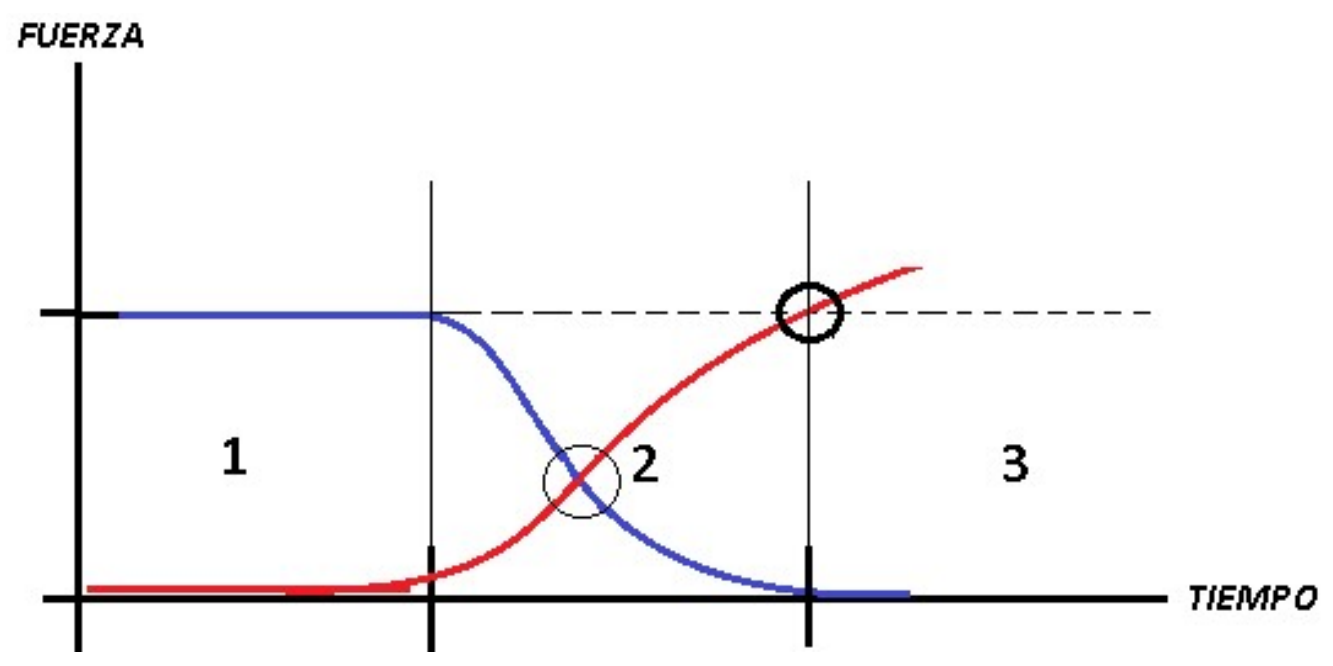

Figura 3. Plano cartesiano indicando las tres fases pertenecientes al modelo de transición estática a dinámica. La línea roja indica la fuerza generada por los tendones de los músculos flexor digital superficial y profundo, la línea azul indica la fuerza generada por el aparato estático pasivo del miembro anterior equino (ligamento suspensorio del menudillo y cabeza carpiana del flexor digital profundo). 1) fase de condición estática; 2) fase de transición (circulo delgado). 3) fase de condición dinámica (circulo grueso)

fuerza ejercida por la tensión de los tendones flexores digitales superficiales $\left(\mathrm{T}_{\mathrm{TFDP}}\right)$ y profundo $\left(\mathrm{T}_{\mathrm{TFDS}}\right)$.

\section{Cálculos de Fuerzas del Biomodelo}

Para determinar la tensión o fuerza mínima necesaria para originar torque en las articulaciones del sistema podotroclear, fue necesario adaptar las fórmulas del modelo de dinámica inversa propuesto por Meershoek y Van den Bogert (2001). Esto permitió identificar la tensión de los tendones flexores $\left(\mathrm{T}_{\text {TFDP }}\right.$ y $\left.\mathrm{T}_{\text {TFDS }}\right)$ y posteriormente establecer la relación de fuerzas necesarias para dar paso a la fase de condición dinámica del biomodelo. Para el desarrollo de estas fórmulas se requirió establecer diferentes brazos de palanca a nivel proximal y distalmente (Figura 4); además del peso calculado para un miembro anterior (PMA) y la fuerza de la reacción de la tierra $(\mathrm{R})$.

Cálculo de los brazos de palanca. Proximalmente, se establecieron tres brazos de palanca. En dos de estos se midió la dis- tancia existente entre el eje metacarpofalángico y los tendones flexores digitales. Del mismo modo se midió la distancia que hay desde el eje de movimiento de dicha articulación hasta el vector perpendicular de R. Distalmente se establecieron dos brazos de palanca. Aquí se midió la distancia existente entre el eje de movimiento de la articulación interfalángica distal y el tendón flexor digital profundo. Además, se midió la distancia que hay entre el eje de movimiento de esta articulación y el vector de R (Figura 4). Las distancias fueron expresadas en milímetros.

Cálculo del peso sobre un miembro anterior (PMA). Según lo establecido por Clayton (2004), el 58\% del peso corporal es soportado por los miembros anteriores. El PMA utilizado correspondió, entonces, al 29\% del peso corporal (100 kg en cada digito anterior).

Cálculo para la fuerza de reacción de la tierra (R). Se expresa en Newtons (N) y se trata de la fuerza que ejerce la tierra con dirección opuesta al peso del animal. Esta fuerza se determinó ubicando el centro de grave- 


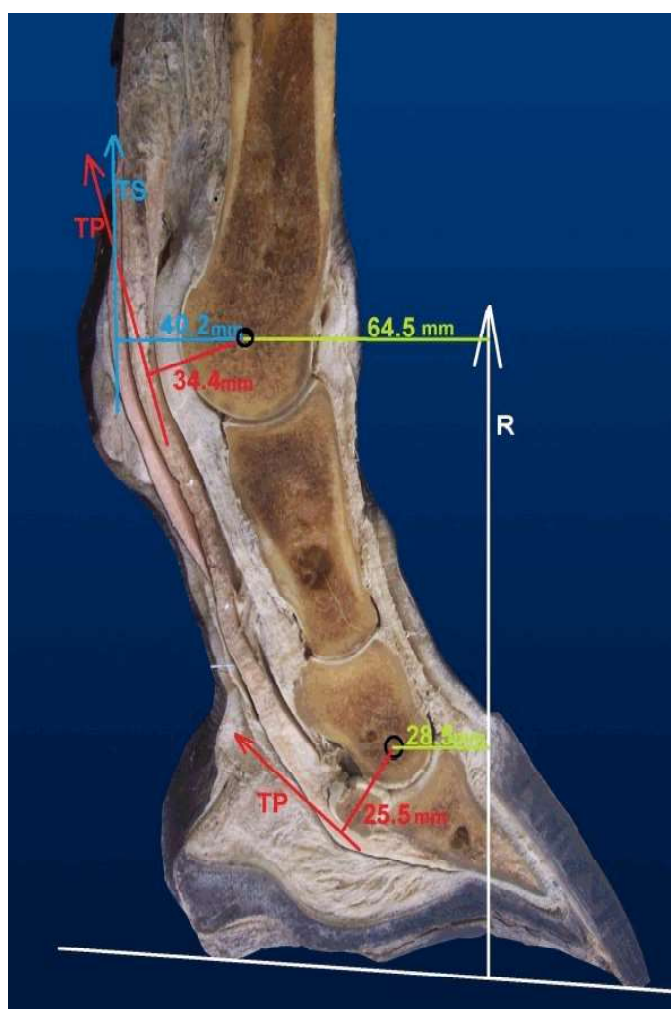

Figura 4. Corte sagital del dígito de un equino. Se muestran mediciones pertenecientes a los brazos de palanca proximal y distalmente (línea azul, líneas rojas y amarillas). También se observa el vector correspondiente a la fuerza de reacción de la tierra $(\mathrm{R})$ (flecha blanca) y los ejes de movimiento en las articulaciones metacarpofalángica e interfalángica distal (círculos negros). Tendón flexor digital profundo (TP) y Tendón flexor digital superficial (TS).

dad vectorial a nivel del rodete coronario y punto de inserción del TFDP de la tercera falange (Figura 4). Para obtener R, se multiplica el PMA por la gravedad $(\mathrm{R}=\mathrm{PMA} \times \mathrm{G})$.

\section{Cálculo de la condición dinámica del} biomodelo. El cálculo del valor de $\mathrm{T}_{\text {TFDP }} \mathrm{se}$ obtuvo multiplicando R por la distancia del brazo de palanca (DBP) de la reacción de la tierra distalmente $\left(\mathrm{DBP}_{\mathrm{R}, \mathrm{DIST}}\right)$ y dividido por la distancia del brazo de palanca del tendón flexor digital profundo distalmente ( $\left.\mathrm{DBP}_{\mathrm{TFDP}, \mathrm{DIST}}\right)$.
$\mathrm{T}_{\text {TFDP }}=\left(\mathrm{R} \times \mathrm{DBP}_{\mathrm{R}, \text { DIST }}\right) / \mathrm{DBP}_{\text {TFDP, DIST }}$

El valor de $\mathrm{T}_{\text {TFDS }}$ se obtuvo al multiplicar R por la DBP de la reacción de la tierra a nivel proximal $\left(\mathrm{DBP}_{\mathrm{R}, \mathrm{PROX}}\right)$, menos el producto de la $\mathrm{T}_{\text {TFDP }}$ por la DBP del tendón flexor digital profundo proximalmente $\left(\mathrm{DBP}_{\mathrm{TFDP}}\right.$, PRox), dividido por la DBP del tendón flexor digital superficial (DBP $\mathrm{DFDS}_{\text {TFS }}$ ).

$$
\begin{gathered}
\mathrm{T}_{\text {TFDS }}=\left(\mathrm{R} \times \mathrm{DBP}_{\mathrm{R}, \text { PROX }}\right)-\left(\mathrm{T}_{\mathrm{TFDP}} \times \mathrm{DBP}_{\mathrm{TFDP},}\right. \\
\text { PROX } \\
\mathrm{DBP}_{\mathrm{TFDS}}
\end{gathered}
$$

\section{Protocolo para el Acondicionamiento de Pruebas Biomecánicas}

Para el acondicionamiento del dedo equino se realizó una disección para exponer los tendones flexores digitales a nivel de la región proximal-palmar del radio. Posteriormente, el dígito se fijó en el prototipo mecánico, tal como se refiere en el apartado respectivo. Durante este proceso de acondicionamiento el material anatómico se fue adaptando a la temperatura ambiente.

\section{Consideraciones Quirúrgicas del Modelo}

Una vez medida la fuerza y tensión del modelo biomecánico en cada dígito, se procedió con la desmotomía bilateral del ligamento suspensorio del hueso navicular (Turner y Mcllwraith, 1982) y se obtuvieron los datos de fuerza. Se registraron 86 datos de fuerza por cada dígito antes de la desmotomía y 86 datos luego de ella. El momento antes de la cirugía se definió como el tiempo prequirúrgico (TPreqx) y el momento después de la cirugía como tiempo posquirúrgico (TPosqx). El intervalo entre cada dato fue de un segundo.

\section{Análisis Estadístico}

Para la evaluación de los resultados de fuerza sobre el hueso navicular por cada dígito se analizó la diferencia estadística existente entre tiempos quirúrgicos (TQX), tanto prequirúrgico (TPreqx) como posquirúrgico (TPosqx) de cada dígito, comparando los promedios de fuerza y límites superior e inferior 


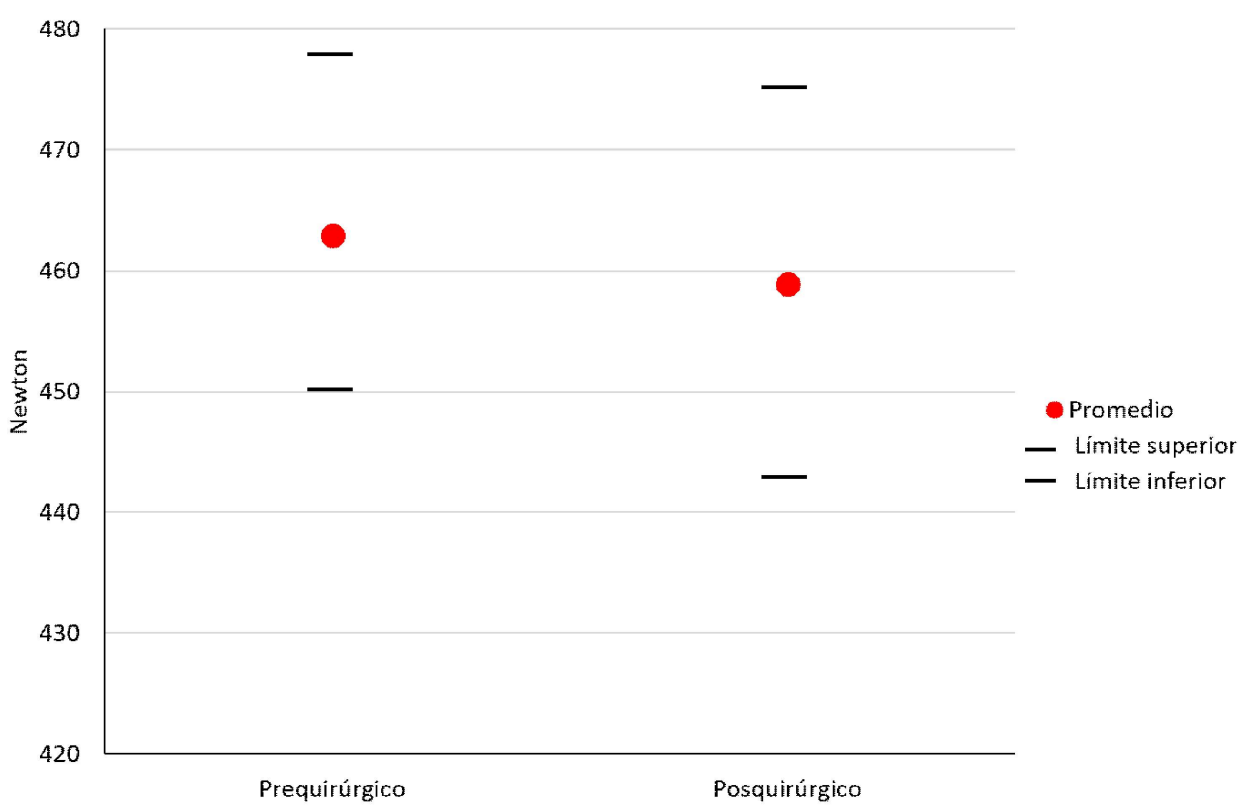

Figura 5. Gráficas de la distribución de fuerza entre los tiempos pre y posquirúrgicos (TQX). Las mediciones observadas equivalen a datos tomados por cada TQX

de cada uno de los dígitos, además de una prueba de $\mathrm{Z}$ calculado con un nivel de confianza del 95\%. Para la evaluación del grado de dispersión y homogeneidad de los datos de fuerza ( 86 datos) de cada dígito, se realizó un análisis de coeficiente de variación, que establece el grado de variabilidad de los datos de cada dígito en su respectivo TQX. Finalmente, para establecer diferencias significativas entre los tiempos quirúrgicos se analizaron los promedios de fuerza y posteriormente una prueba de $\mathrm{Z}$ calculado.

\section{Resultados y Discusión}

Las fuerzas medidas sobre el hueso navicular de cada dígito presentaron escasa variabilidad, tanto en TPreqx como TPosqx. Esto indicó una distribución de datos más homogénea por dígito, y una obtención de datos metodológicamente adecuada. No obstante, algunos dígitos $(3,4,6)$ presentaron mayor variabilidad de los TQX entre los promedios de fuerza y Z calculado (Cuadro 1). Estos resultados indican que en la mayoría de los casos, el modelo biomecánico se ajustaba de forma controlada para la evaluación en conjunto del efecto de la desmotomía y presentaba pocas variaciones asociadas a efectos externos o de manipulación de las fuerzas.

Las variaciones individuales que se dieron en los dígitos 3, 4 y 6 pueden explicarse por la variabilidad biológica de los tendones flexores y falta de precisión de los componentes electrónicos de medición. La actividad biológica y el potencial de energía elástica de los tendones son, además, factores que cambian con la edad del animal y su actividad física, los que a su vez pueden influenciar directamente en los enlaces cruzados del colágeno de los tendones disminuyendo su resistencia a la tracción (Nordin y Frankel, 2013). Otra posible causa de variabilidad de las fuerzas se puede atribuir al funcionamiento 
Cuadro 1. Descripción de los promedios de fuerza para los tiempos quirúrgicos (TQXs), límite superior (L.S.) e inferior (L.I.), coeficiente de variación (CV) y Newtons por kilogramo de peso vivo de dígitos de equinos utilizando un modelo biomecánico

\begin{tabular}{ccccccccccc}
\hline & \multicolumn{3}{c}{ Tiempo prequirúrgico } & \multicolumn{6}{c}{ Tiempo posquirúrgico } & \\
\cline { 2 - 10 } & $\begin{array}{c}\text { Newton } \\
\text { promedio }\end{array}$ & L.I. & L.S. & $\begin{array}{c}\text { CV } \\
\%\end{array}$ & $\begin{array}{c}\text { Newton } \\
/ \mathrm{kg}\end{array}$ & $\begin{array}{c}\text { Newton } \\
\text { promedio }\end{array}$ & L.I. & L.S. & $\begin{array}{c}\mathrm{CV} \\
\%\end{array}$ & $\mathrm{Z}$ \\
\hline 1 & 451.3 & 446.7 & 456.0 & 1.0 & 4.5 & 444.0 & 438.4 & 447.6 & 1.0 & 1.29 \\
2 & 463.7 & 460.9 & 466.5 & 0.6 & 4.6 & 468.7 & 462.6 & 474.9 & 1.3 & -0.74 \\
3 & $397.5^{1}$ & 392.4 & 402.5 & 1.3 & 4.0 & $383.5^{1}$ & 378.9 & 388.1 & 1.2 & $2.05^{2}$ \\
4 & $498.7^{1}$ & 496.3 & 501.2 & 0.5 & 5.0 & $526.5^{1}$ & 522.8 & 530.3 & 0.7 & $6.18^{2}$ \\
5 & 439.4 & 431.1 & 447.6 & 1.9 & 4.4 & 428.6 & 420.9 & 436.3 & 1.8 & 0.95 \\
6 & $430.8^{1}$ & 424.3 & 437.3 & 1.5 & 4.3 & $405.0^{1}$ & 400.7 & 409.3 & 1.1 & $3.31^{2}$ \\
7 & 456.4 & 453.9 & 458.9 & 0.5 & 4.6 & 451.5 & 448.6 & 454.4 & 0.6 & 1.28 \\
8 & $553.1^{3}$ & 544.9 & 561.4 & 1.5 & $5.5^{3}$ & $541.3^{3}$ & 533.2 & 549.40 & 1.5 & 1.01 \\
9 & 454.6 & 451.0 & 458.2 & 0.8 & 4.5 & 459.0 & 454.5 & 463.4 & 1.0 & -0.75 \\
10 & 488.2 & 484.6 & 491.9 & 0.8 & 4.9 & 483.3 & 479.3 & 487.2 & 0.8 & 0.92 \\
\hline
\end{tabular}

del sensor, componente que puede presentar un error de precisión en la linealidad del 3\% aproximadamente (Tekscan, s.f.).

En el análisis de fuerza entre ambos TQX se observó una igualdad entre los intervalos de los tiempos, lo cual indica igualdad estadística de los promedios de fuerza para los TQX, con una diferencia entre tiempos quirúrgicos menor al $1 \%$ (Figura 5). El valor de $\mathrm{Z}$ calculado hallado entre los intervalos de confianza establecidos, indicó una igualdad estadística de las mediciones de fuerza entre TPreqx y TPosqx y una diferencia entre dichos tiempos de $0.95 \%$ (4.4 Newton) indicando que la desmotomía de los ligamentos suspensorios del hueso navicular con el eje podofalángico a $45^{\circ}$ generaba escasos cambios del comportamiento biomecánico en el hueso navicular.
No se encontraron estudios semejantes in vitro para comparar los resultados obtenidos. No obstante, se debe tener presente que en estudios realizados in vivo y con el animal al trote se reportaron fuerzas soportadas por el hueso navicular similares a las encontradas en este estudio. Wilson y McGuigan (2001) determinaron in vivo una fuerza máxima de 5.62 Newton $/ \mathrm{kg}$ de peso vivo en caballos purasangre, en tanto que Willemen et al. (1999) determinó una fuerza máxima de 6 Newton/kg de peso vivo. Esto evidencia una diferencia de la fuerza máxima de 0.12 y 0.5 Newtons $/ \mathrm{kg}$ para el primer y segundo reporte en comparación con la máxima fuerza registrada en el presente estudio (Cuadro 1). Este resultado indica que desde el punto de vista biomecánico en el presente estudio in vitro se aproxima considerablemente a variables físicas in vivo como lo es el potencial de energía elástica del tendón flexor digital 
profundo durante el trote y su consecuente fuerza sobre el hueso navicular.

\section{Conclusiones}

La desmotomía de los ligamentos suspensorios del navicular realizada en condiciones in vitro y con eje podofalángico a $45^{\circ}$ no originó cambios significativos de fuerza sobre el hueso navicular.

\section{Literatura Citada}

1. Back W, Clayton H. 2013. Equine locomotion, $2^{\text {nd }}$ ed. Edinburg, UK: Elsevier. $528 \mathrm{p}$.

2. Bell BT, Bridge IS, Sullivan ST. 1996. Surgical treatment of navicular syndrome in the horse using navicular suspensory desmotomy. New Z Vet J 44: 26-30. doi: 10.1080/00480169.1996.35927

3. Becker CK, Savelberg HH, Buchner HH, Barneveld A. 1998. Long-term consequences of experimental desmotomy of the accessory ligament of the deep digital flexor tendon in adult horses. Am J Vet Res 59: 347-351.

4. Buchner HF, Savelberg HH, Becker CK. 1996. Load redistribution after desmotomy of the accessory ligament of the deep digital flexor tendon in adult horses. Vet Quart 18: 70-74. doi: 10.1080/ 01652176.1996.9694697

5. Clayton HM. 2004. The dynamic horse. Michigan, USA: Sport Horse Publications. $267 \mathrm{p}$.

6. Meershoek LS, van den Bogert AJ, Schamhardt HC. 2001. Model formulation and determination of in vitro parameters of a noninvasive method to calculate flexor tendon forces in the equine forelimb. Am J Vet Res 62: 15851593. doi: 10.2460/ajvr.2001.62.1585
7. Nordin M, Frankel V. 2013. Bases biomecánicas del sistema músculo esquelético. $4^{\circ}$ ed. España: Lippincott Willliams \& Wilkins. $113 \mathrm{p}$.

8. Pool RR, Meagher DM, Stover SM. 1989. Pathophysiology of navicular disease. Vet Clin N Am Equine 5: 109129. doi: 10.1016/S0749-0739(17)30606-5

9. Stashak TS. 2003. Adams: claudicación en el caballo. $5^{\circ}$ ed. Buenos Aires: InterMedica. $264 \mathrm{p}$.

10. Tekscan (sf). Pressure mapping, force measurement \& tactile sensors. [Internet]. Disponible en: www. tekscan.com

11. Turner SA, McIlwraith WC. 1982. Técnicas quirúrgicas en animales grandes. Buenos Aires: Ed. Hemisferio Sur. $343 \mathrm{p}$.

12. Watkins JP, McMullan WN, Morris EL. 1993. Navicular suspensory desmotomy in the management of navicular syndrome: a retrospective analysis. Am Assoc Equine Pract 39: 261-262.

13. Willemen MA, Savelberg HH, Barneveld A. 1999. The effect of orthopaedic shoeing on the force exerted by the deep digital flexor tendon on the navicular bone in horses. Equine Vet $\mathrm{J}$ 31: 25-30. doi: 10.1111/j.20423306.1999.tb03787.x

14. Wilson AM, McGuigan MP, Fouracre L, MacMahon L. 2001. The force and contact stress on the navicular bone during trot locomotion in sound horses and horses with navicular disease. Equine Vet J 33: 159-165. doi: 10.1111/j.20423306.2001.tb00594.x

15. Wright IM. 1986. Navicular suspensory desmotomy in the treatment of navicular disease: technique and preliminary results. Equine Vet J 18: 443-446.

16. Wright IM. 1993. A study of 118 cases of navicular disease: treatment by navicular suspensory desmotomy. Equine Vet J 25: 501-509. doi: 10.1111/j.20423306.1993.tb03001.x 